\title{
Neonatal Immunization: Rationale, Current State, and Future Prospects
}

\author{
Elizabeth Whittaker ${ }^{1}$, David Goldblatt ${ }^{2}$, Peter McIntyre ${ }^{3}$ and Ofer Levy ${ }^{4,5 *}$ \\ ${ }^{1}$ Centre for International Child Health, Department of Paediatrics, Imperial College London, London, United Kingdom, \\ 2Immunobiology Section, UCL Great Ormond Street Institute of Child Health (ICH), London, United Kingdom, ${ }^{3}$ National \\ Centre for Immunisation Research and Surveillance, Kids Research, Sydney Children's Hospital Network and University of \\ Sydney, Sydney, NSW, Australia, 'Precision Vaccines Program, Division of Infectious Diseases, Boston Children's Hospital, \\ Boston, MA, United States, ${ }^{5}$ Harvard Medical School, Boston, MA, United States
}

OPEN ACCESS

Edited by:

Fabio Bagnoli,

GlaxoSmithKline (Italy), Italy

Reviewed by:

Robert Cohen,

Centre Hospitalier

intercommunal de Créteil,

France

Katie Louise Flanagan,

RMIT University, Australia

${ }^{*}$ Correspondence:

Ofer Levy

ofer.levy@childrens.harvard.edu

Specialty section:

This article was submitted to

Vaccines and Molecular

Therapeutics,

a section of the journal

Frontiers in Immunology

Received: 13 October 2017

Accepted: 28 February 2018

Published: 04 April 2018

Citation:

Whittaker E, Goldblatt D, Mclntyre P

and Levy O (2018) Neonatal

Immunization: Rationale, Current

State, and Future Prospects.

Front. Immunol. 9:532.

doi: 10.3389/fimmu.2018.00532
Infections take their greatest toll in early life necessitating robust approaches to protect the very young. Here, we review the rationale, current state, and future research directions for one such approach: neonatal immunization. Challenges to neonatal immunization include natural concern about safety as well as a distinct neonatal immune system that is generally polarized against Th1 responses to many stimuli such that some vaccines that are effective in adults are not in newborns. Nevertheless, neonatal immunization could result in high-population penetration as birth is a reliable point of healthcare contact, and offers an opportunity for early protection of the young, including preterm newborns who are deficient in maternal antibodies. Despite distinct immunity and reduced responses to some vaccines, several vaccines have proven safe and effective at birth. While some vaccines such as polysaccharide vaccines have little effectiveness at birth, hepatitis $B$ vaccine can prime at birth and requires multiple doses to achieve protection, whereas the live-attenuated Bacille Calmette-Guérin (BCG), may offer single shot protection, potentially in part via heterologous ("non-specific") beneficial effects. Additional vaccines have been studied at birth including those directed against pertussis, pneumococcus, Haemophilus influenza type B and rotavirus providing important lessons. Current areas of research in neonatal vaccinology include characterization of early life immune ontogeny, heterogeneity in and heterologous effects of BCG vaccine formulations, applying systems biology and systems serology, in vitro platforms that model age-specific human immunity and discovery and development of novel age-specific adjuvantation systems. These approaches may inform, de-risk, and accelerate development of novel vaccines for use in early life. Key stakeholders, including the general public, should be engaged in assessing the opportunities and challenges inherent to neonatal immunization.

Keywords: neonatal, vaccine, protection, trained immunity, novel adjuvants

\section{INTRODUCTION}

Despite the success of the Millenium Development Goal era from 2000 to 2015, during which the under five mortality rate was reduced by 53\%, 2 million infants under 6 months die annually due to infections (1). Of the 5.9 million children under 5 years of age who died in $2015,45 \%$ were in the first month of life (2). Many of these deaths are attributed to vaccine preventable illnesses, occurring before protection is afforded by routine immunization given as part of the expanded program of immunization (EPI). Although this commences at 6-8 weeks of age, the first dose does not provide 
immediate protection and multiple doses are required, leading to vulnerability in the first 6 months of life. In an effort to reduce the under 5-year old mortality rate further, to $\leq 25 / 1,000$ live births by the end of 2030, a number of strategies are being explored and implemented as part of the sustainable development goal-3. These include maternal immunization, which, although it shows great promise for a number of pathogens, including pertussis and influenza, is limited by safety and ethical concerns, and is of limited value for the $\sim 2.6$ million infants born preterm, prior to maternal antibody (Ab) transfer (3). The reality, that we rely on immunization occurring early in life, coupled with recent advances in our understanding of neonatal immune responses (4-6), has led to renewed interest in neonatal immunization as a promising and effective strategy, to reduce morbidity and mortality in young infants. Thus, the topic of early life immunity, and in particular neonatal immunization, is one of tremendous public health relevance.

Great strides in vaccine development over the last century have resulted in a number of effective vaccines being given in early life, but only Bacille Calmette-Guérin (BCG), hepatitis $\mathrm{B}(\mathrm{HBV})$, and polio vaccine [oral polio vaccine (OPV); or inactivated polio vaccine (IPV)] have been routinely recommended at birth. For some pathogens, including pertussis and tuberculosis (TB), better vaccines are needed, while for others such as human immunodeficiency virus (HIV) and respiratory syncytial virus (RSV), efficacious vaccines have yet to be developed and licensed for any age group. Among the approaches to improving protection against infection in early life, neonatal immunization is ripe for further research and development. Herein, we review the rationale for neonatal immunization and highlight essential research areas, including the study of immune ontogeny and the development of vaccines optimized for early life administration.

\section{Rationale for Use of Vaccines in the Neonatal Period}

The neonatal period is defined as the first 28 days of life. For the purpose of this review, we define neonatal vaccines as those given "at birth" or within the first 28 days of life. Of note, EPI vaccines are licensed to be given within the first few weeks of life, and in reality, the "birth dose" is given across a range of time in the first month of life, a variability that to our knowledge has not been systematically studied with respect to relative vaccine efficacy. In contrast, we define infant vaccines as those given after the first 28 days of life. In countries following the EPI schedule, after the neonatal doses of BCG, HBV, and polio vaccines, the next EPI schedule dose is typically given between 6 and 8 weeks of life. As with any vaccine approach, development of neonatal vaccines must take into account potential limitations, including: (a) need to establish safety, (b) lack of effectiveness of some vaccines in early life, (c) challenges of a translational path that typically starts with formulations optimized for adults, rather than generating formulations that are optimal for the young, and (d) potential blunting of neonatal $\mathrm{Ab}$ responses after maternal immunization. Nevertheless, the rationale for neonatal immunization is robust and includes: (a) the heavy burden of early life infection; (b) that birth is a practical point of healthcare contact, and pairing immunization with birth may lead to health benefits for both mothers and newborns; (c) immunization at birth may provide earlier protection than existing immunization schedules; (d) the likely benefit of protection to babies born preterm for whom maternal $\mathrm{Ab}$ transfer is limited, with an increased risk of serious infections throughout childhood (7); and (e) emerging evidence that the heterologous benefit of the live-attenuated BCG vaccine and other live vaccines may be greatest in early life (8).

\section{LESSONS FROM IMMUNE ONTOGENY}

Neonatal immunization occurs in a backdrop of distinct early life immunity. Recent reviews have highlighted that both cellular and soluble aspects of the immune system are distinct at birth $(9,10)$. Neonatal immunity must not only defend the newborn against a potential onslaught of potential pathogens, but also mediate the acquisition of a colonizing microbiome over the first hours and days of life. In this context, neonatal immune responses are apparently designed to avoid excessive inflammation with a generally reduced production of pro-inflammatory and Th1-polarizing cytokines to microbial components/pattern recognition receptors (PRR) agonists. Age-specific composition of soluble and cellular factors shape neonatal immunity. The distinct composition of human newborn cord blood plasma includes soluble mediators such as maternal Abs, high levels of immunosuppressive adenosine, and low levels of complement, important for triggering adaptive immune responses (11). Accordingly, modeling age-specific immunity in vitro should take into account distinct composition of age-specific autologous plasma, rather than, for example, fetal bovine serum (9). Distinct cellular immunity in the newborn includes reduced Th1 but robust anti-inflammatory IL-10 responses of antigen-presenting cells to stimulation by PRR agonists, high frequency of naïve- and regulatory-T cells and CD71+ erythroid precursors that may limit, for example, responses to pertussis immunization $(10,12,13)$. Nevertheless, neonatal immunity is capable of mounting antigen-specific effector responses, as demonstrated by BCG-specific IFN $\gamma$ production following vaccination at birth (14). Overall, detailed study and modeling of age-specific human immunity may help inform development of vaccine formulations, with or without adjuvants as needed, that may trigger a protective immune response in early life.

\section{PROOF OF CONCEPT: ROUTINE NEONATAL VACCINES}

\section{Bacille Calmette-Guérin}

Bacille Calmette-Guérin is a live-attenuated strain of Mycobacterium bovis. Given in areas with high-endemic TB to prevent disseminated TB in infancy, BCG is the most commonly given vaccine with $\sim 4$ billion doses administered to date. Although it has been administered for nearly 100 years, several key issues regarding BCG have emerged, including: (a) lack of a clear correlate of protection (CoP); (b) marked heterogeneity 
between licensed BCG formulations (15); and (c) growing evidence that BCG has heterologous ("non-specific") beneficial effects, particularly when administered in newborns (16). It is hypothesized that these non-specific benefits may protect against unrelated infections, supporting the use in neonates, beyond any protection against TB infection or disease. Furthermore, association studies suggest that early immunization with BCGcontaining regimens may protect against leukemia, allergy, and childhood diabetes among others, possibly via heterologous trained immunity (17-19).

A CoP is an immune measure that corresponds to vaccineinduced protection from disease (20). Despite substantial efforts to characterize classic adaptive immunity, including multiple studies of polyfunctional CD4 T cells, a clear CoP for BCG has yet to be established (21). Indeed, increasing evidence that BCG induces trained immunity-i.e., enhanced subsequent innate responses to a range of stimuli (8)-raises the possibility that these innate immune enhancing effects may not only underlie heterologous (non-specific) benefits of BCG vaccine, but may also contribute to, or conceivably be, the major factor in the so called "specific" effect of BCG, i.e., protection against early life TB.

A critical issue with respect to BCG immunization is marked variability between vaccine formulations produced in different production facilities, with the result that "BCG" is not a single entity. After its original manufacture in the Pasteur Institute (Paris, France) in 1921, BCG was shipped to 20 different international sites where the vaccine was repeatedly subcultured under different conditions. This has resulted in diverse licensed BCG formulations that are distinct both by content of live mycobacteria as well as genetic composition. These strains have been shown to have differing immune responses and furthermore, the clinical relevance of this has been illustrated in comparative studies, which suggest, for example, that BCG-Denmark and BCG-Japan may have greater benefit in reducing TB disease than BCG-Russia $(15,22)$.

Much remains to be learned regarding mechanisms underlying BCG-induced protection. As a complex vaccine comprised of live-attenuated mycobacterium, BCG engages the innate immune system via PRRs. Analogy to M. tuberculosis as well as direct human in vitro studies suggests that BCG may activate via multiple toll-like receptors (TLRs) including TLRs-2, -4, -7, -8, and -9 as well as C-type lectin receptors and NLRs (23). Studies of BCG-immunized adults demonstrate a re-programing of monocyte precursors such that the higher expression of PRRs and greater reactivity to stimuli such as TLR agonists. This innate immune enhancing effect of BCG is reminiscent of the effect of administration of TLR agonists to neonatal mice that enhanced innate immune responses, including cytokine induction and phagocyte recruitment, and improved bacterial clearance and survival in a model of neonatal sepsis (24). Such innate immune enhancing effects of prior stimulation have been termed "trained immunity" reflecting an adaptive arm of innate immunity that is noted in plants, insects, and mammals (25). That BCG heterologous (non-specific) benefits are greater in early life (26) suggests ontogeny of underlying immune mechanisms, potentially including trained immunity (27).

\section{Hepatitis B Vaccine}

Hepatitis B vaccine is an alum-adjuvanted vaccine containing hepatitis B surface antigen (HBsAg). The alum-adjuvanted HBV is given within the EPI (Table 1) and also in Australia, Europe, and United States, where a birth dose is recommended (28). With respect to innate immune activation, while the Alum adjuvant present in $\mathrm{HBV}$ may engage the inflammasome, HBsAg also interacts with CD14 to activate dendritic cells (29). Importantly, there is a measurable $\mathrm{CoP}$ for $\mathrm{HBV}$, namely the titer of anti-HBsAg Abs. Although this CoP has been defined, as with many vaccines, it is not fully understood how $\mathrm{HBV}$ vaccine induces protective immunity, as a newborn dose is protective, despite only 30-50\% of newborns responding with "protective" titers after a single dose. This observation suggests that additional mechanisms, including cell-mediated immunity (CMI), may contribute to protection (30). Ab and T cell responses to HBV given to infants are distinct from those of adults, in that infants produced markedly higher serum anti-hepatitis B surface (HBs) Ab titers in one study, and low-Ab levels were associated with lower HBs Ag-specific IFN $\gamma$ responses and a more Th2-polarized memory response to $\mathrm{HBs} \mathrm{Ag}$

TABLE 1 | Immunizations given at different ages

\begin{tabular}{|c|c|c|c|}
\hline & $\begin{array}{l}\text { Vaccines } \\
\text { licensed }\end{array}$ & Vaccines tested & $\begin{array}{l}\text { Future vaccine } \\
\text { targets }\end{array}$ \\
\hline Pregnancy & $\begin{array}{l}\text { - } \text { aPertussis } \\
\text { - Tetanus } \\
\text { - Influenza }\end{array}$ & $\begin{array}{l}\text { - }(\mathrm{RSV}) \\
\text { - } \mathrm{GBS}\end{array}$ & $\begin{array}{ll}\text { - } & \text { Group B } \\
& \text { Streptococcus } \\
\text { - } & \text { HIV } \\
\text { - } & \text { Malaria } \\
\end{array}$ \\
\hline Birth & $\begin{array}{l}\text { - } \text { OPV } \\
\text { - } \mathrm{HepB} \\
\text { - } \text { BCG }\end{array}$ & $\begin{aligned} & \text { - } \text { DTaP } \\
& \text { - } \text { Hib } \\
& \text { - } \text { PCV } \\
& \text { - } \text { Malaria (e.g., } \\
& \text { RTS,S/ASO1/2) } \\
& \text { - } \text { Recombinant } \\
& \text { BCG vaccines } \\
& \text { (e.g., VPM1002) } \\
& \text { - } \text { HIV (phase I/lla) } \\
& \text { - } \text { Rotavirus } \\
&\end{aligned}$ & $\begin{array}{l}\text { - } \mathrm{RSV} \\
\text { - } \text { Salmonella } \\
\text { - } \mathrm{ETEC} \\
\text { - } \mathrm{ncHl} \\
\text { - } \text { Malaria }\end{array}$ \\
\hline $\begin{array}{l}\text { Infant doses } \\
\text { Age 2-4 months }\end{array}$ & $\begin{array}{l}\text { - } \text { DTaP and } \\
\text { DTwP } \\
\text { - } \mathrm{IPV} \\
\text { - Hib } \\
\text { - HepB } \\
\text { - } \mathrm{PCV} \\
\text { - MenB } \\
\text { - MenC } \\
\text { - Rotavirus }\end{array}$ & $\begin{array}{l}\text { - Malaria (e.g., RTS, } \\
\text { S/ASO1/2, and } \\
\text { Spf66) } \\
\text { - } \text { Recombinant BCG } \\
\text { vaccines } \\
\text { (e.g., VPM1002) } \\
\text { - } \text { Novel TB candidates } \\
\text { (e.g., MVA85A) } \\
\text { - HIV (phase I/lla) }\end{array}$ & $\begin{array}{l}\text { - } \text { RSV } \\
\text { - } \text { Men ACWY } \\
\text { - Salmonella } \\
\text { - } \text { ETEC } \\
\text { - } \text { ncHI } \\
\text { - } \text { Malaria }\end{array}$ \\
\hline $\begin{array}{l}\text { Infant doses } \\
\text { Age 12-13 months }\end{array}$ & $\begin{array}{l}\text { - } \text { Hib } \\
\text { - } \mathrm{PCV} \\
\text { - } \mathrm{MMR} \\
\text { - } \mathrm{MenB} \\
\text { - MenC } \\
\text { - } \text { Varicella }\end{array}$ & - LAIV & \\
\hline
\end{tabular}

RSV (respiratory syncytial virus), Hib (haemophilus influenzae B), BCG (Bacille CalmetteGuerin), OPV (oral polio vaccine), IPV (inactivated polio vaccine), HepB (hepatitis B), DTaP (diphtheria, tetanus, acellular pertussis), DTwP (diphtheria, tetanus, whole cell pertussis), PCV (pneumococcal conjugate vaccine), HIV (human immunodeficiency virus), ETEC (enterotoxic Escherichia coli), ncHI (non-encapsulated Haemophilus influenzae), Men B,C ACWY (meningococcal B,C, ACWY), MMR (measles, mumps, rubella), TB (tuberculosis), LAIV (live-attenuated influenza virus). () indicates Trial in progress. 
at 1 year (30). Genetic factors, low-birth weight and low-Apgar scores were risk factors for poor HBV response in a study of twins in China (31). Haplotype analysis of Gambian infants suggested that CDC42, IL19, and IL1R1 genes associated with peak antiHBsAg Ab level (32). Much remains to be learned regarding how $\mathrm{HBV}$ protects in early life.

\section{Polio}

A birth dose of OPV has been recommended by the World Health Organization since 1984. It is hypothesized that a birth dose of OPV may induce mucosal protection prior to colonization or infection with enteric organisms which may interfere with the immune response to doses given later in life. Data on seroconversion following this individual dose of trivalent OPV (tOPV) vary greatly, from 10 to $15 \%$ in India to $76 \%$ in South Africa, however, the positive impact on levels of neutralizing Abs and seroconversion rates on completion of the routine immunization schedule are undisputed (33). A systematic review of 5,257 infants given tOPV at birth, found that the percentage of newborns who seroconverted at 8 weeks ranged between 6 and 42\% for poliovirus type 1, 2 and $63 \%$ for type 2 , and 1 and $35 \%$ for type 3 (34). In addition, there were four studies of IPV in newborns with a seroconversion rate of $8-100 \%$ for serotype $1,15-100 \%$ for serotype 2 , and $15-94 \%$ for serotype 3, measured at 4-6 weeks of life. No serious adverse events related to OPV or IPV doses at birth were reported in these studies, including no cases of acute flaccid paralysis. Some groups have advocated a shift to using IPV because (a) tOPV has been associated with rare cases of vaccine-associated paralytic poliomyelitis ( 2-4 cases/million), (b) concerns about the use of live vaccines in immunocompromised individuals, including those with HIV infection, and (c) potential risk of strain reversion. Of note, however, some studies have suggested that similarly to BCG vaccine, a birth dose of live OPV may induce heterologous ("nonspecific") beneficial effects (35). Further research is warranted prior to replacing OPV with IPV (36).

\section{CLINICAL STUDIES OF OTHER VACCINES AT BIRTH}

\section{Pertussis}

Initial studies of the role of a birth dose of whole cell pertussis vaccine demonstrated low-Ab titers at 4 months, although there were no randomized studies at that time comparing a birth dose with a dose at 6-8 weeks of age (37). Further studies of neonatal whole cell pertussis immunization were deterred by the suggestion in 1965 that immunization with the whole cell pertussis vaccine combined with diphtheria and tetanus toxoids (DTwP) within $24 \mathrm{~h}$ of birth may introduce "immune paralysis" (38). Twenty years later, comparison studies of a birth dose of DTP with routine immunization at 2 months demonstrated significantly lower titers to pertussis toxin (PT) at 9 months of age, and an inverse correlation between cord Ab titers and infant responses (39). Safety concerns about the whole cell vaccine led to a switch to acellular pertussis vaccines (aP, or DTaP) in the 1990s. Initial neonatal studies of aP vaccines, both with and without diphtheria and tetanus antigens, were promising
$(40,41)$, but a conflicting later study showed poorer responses (42). Reports of bystander interference resulting in lower Haemophilus influenza type B (Hib) vaccine and HBV responses were concerning (41). A pilot study of the GSK monovalent aP vaccine at birth and 4 weeks demonstrated significantly higher IgG $\mathrm{Ab}$ against pertussis antigens at 2 months of age, without reducing subsequent pertussis $\mathrm{Ab}$ responses. A larger study of doses at birth and 6 weeks, including influence of maternal immunization, on $\mathrm{Ab}$ responses up to 5 years of age is ongoing $(43,44)$. Follow-up of children from the initial pilot study to 4 years of age demonstrated higher cytokine responses to pertussis antigen stimulation in those who received a birth dose compared with controls at 2 years of age (44). These observations were similar to those in a long-term follow-up study of children vaccinated at birth which showed increasing CMI, as measured by lymphoproliferative capacity, compared with controls (45). As the role of maternal immunization with pertussis becomes more established, it is crucial to include the effect of maternal interference in studies, as even pre-pregnancy immunization may influence later-born infant responses (46). Englund et al. demonstrated a lack of maternal $\mathrm{Ab}$ interference on infant immunizations given at 2 months - that is, the PT Ab response to DTaP, unlike DTwP, was not affected by pre-existing Ab to PT (47). Whether this observation also holds true for birth doses requires future study. Given current concerns of waning immunity to aP (48), novel pertussis vaccine formulations, potentially including developing pertussis vaccines that are safe and effective in newborns, are needed to induce robust and durable immunity against this pathogen.

\section{Haemophilus Influenza Type B}

The role of a birth dose of Hib vaccine was explored by a number of groups following the initial success of its introduction into the EPI. Three different conjugate vaccines tested \{HIB polysaccharide conjugated to tetanus toxoid, Hib polysaccharide conjugated to a genetically modified diphtheria toxin ( $\mathrm{HbOC}$ ), and Hib polysaccharide conjugated to a Neisseria meningitidis outer membrane protein [HbOMP; subsequently noted to be a TLR2 agonist (49)]\} all resulted in significantly higher PRP Ab levels at 2 or 4 months compared with controls, suggesting neonatal priming was possible $(50,51)$. However, these higher Ab levels did not persist for $\mathrm{HbOC}$ and declined more rapidly than controls for HbOMP, leading to concerns about waning protection. Further studies have not been undertaken, as epidemiological studies have demonstrated a protective effect of herd immunity on early life burden of invasive Hib disease, presumed due to reduction of asymptomatic nasopharyngeal carriage of Hib among vaccines $(52,53)$. Conjugate vaccines, through a T cell-dependent immune response, result in very high-protective $\mathrm{Ab}$ responses in infants of all ages, which results in reduction of carriage. Furthermore, the licensed 10 valent pneumococcal conjugate vaccine (PCV10; PHid-CV), uses a Haemophilus outer membrane protein (protein D) as its carrier protein, and immunization with this reduces the incidence of all invasive Haemophilus spp. disease, including non-typable or non-encapsulated Haemophilus influenzae (ncHI). This may be of particular importance as invasive disease due to $\mathrm{ncHI}$ is commonest in the first month of life $(54,55)$, so the 
potential role of the PHid-CV pneumococcal vaccine or a specific ncHI vaccine in neonates should be studied.

\section{Pneumococcal Conjugate Vaccine}

To date, only two published trials have assessed effects of a neonatal dose of the seven valent PCV7 (56, 57). In Kenya, 300 neonates were randomized to receive PCV7 at birth, 10 and 14 weeks or at 6,10 , and 14 weeks (EPI schedule). The safety of giving vaccine at birth was an important endpoint in the study and the researchers saw no significant difference in safety events between the two groups. Serotype-specific IgG binding was measured following the completion of the primary infant schedule at 18 weeks of age. The proportion of infants who had an Ab concentration above the accepted protective threshold of $>0.35 \mu \mathrm{g} / \mathrm{mL}$ was similar between the two groups. When a higher threshold $(>1.0 \mu \mathrm{g} / \mathrm{mL})$ was used, proportions above 1 for serotypes $4,18 \mathrm{C}, 19 \mathrm{~F}$ were lower in the neonatal group. Geometric mean concentrations of IgG for four serotypes $(4,9 \mathrm{~V}, 18 \mathrm{C}, 19 \mathrm{~F})$ were lower in the neonatal group compared with the EPI group at 18 weeks of age. In contrast, the mean avidity indices were significantly higher in the neonatal group for three of the four serotypes tested (4, $6 \mathrm{~B}, 19 \mathrm{~F}$ ) (57). In both groups, maternal IgG measured in cord blood inversely correlated with the GMC at 18 weeks of age with high-serotype specific cord blood levels associated with lower responses to vaccine. At 9 months of age, 5 months following the third PCV dose, there was no difference in the percentage of infants with an $\mathrm{Ab}$ concentration above either the 0.35 or $1.0 \mu \mathrm{g} /$ $\mathrm{mL}$ thresholds. The GMC of serotype 4 specific IgG remained lower and serotype $19 \mathrm{~F}$ avidity index remained higher in the neonatal group. Responses to a booster dose of PCV given at 9 months of age and measured 2 weeks later were comparable between the two groups suggesting that there is no tolerance induced by the neonatal dose. Carriage was measured at 18 and 36 weeks in this study with no significant differences detected between the groups.

In a trial undertaken in Papua New Guinea (PNG), 318 infants were randomized to receive either PCV7 at birth, 1 and 2 months, PCV7 at 1-3 months, or no PCV7 (56). Local reactogenicity rates were generally low although higher rates were seen in the infant than the neonatal group. There were no differences in the illness episodes or serious adverse events. At 2 months of age, serotypespecific GMCs were significantly higher in the neonatal group than in the infant group for four of the seven serotypes in PCV7 $(4,9 \mathrm{~V}, 18 \mathrm{C}, 19 \mathrm{~F})$. At this point, the neonatal group had received two doses of PCV compared with one dose in the infant group. By 4 months of age, following three doses of the vaccine, GMCs were significantly higher for all serotypes in the infant group than in the neonatal group although 2 months had elapsed since the neonatal groups third dose, a gap that was only 1 month for the infant group. Comparable responses were seen following a pneumococcal polysaccharide vaccine administered at 9 months of age and responses in both PCV7 primed groups were significantly greater than responses in those who were not primed. Nasopharyngeal swabs were collected at ages 1-4 weeks and 3, 9, 18 months, and middle ear discharge if present. The prevalence of pneumococcal carriage was $22 \%$ at 1 week of age, rising to $80 \%$ by age 3 months and remained $>70 \%$ thereafter (58). There were no significant differences in PCV7 serotype carriage between PCV recipients and controls at any age ( 22 vs. $31 \%$ at 9 months, $p=0.2$ ). At age 9 months, the prevalence of non-PCV7 serotype carriage was $17 \%$ higher in PCV7 recipients $(48 \%)$ than in controls $(25 \%, p=0.02)$. The authors attributed the limited impact of neonatal or accelerated infant PCV7 schedules on vaccine serotype carriage to the early onset of dense carriage of a broad range of pneumococcal serotypes.

A prior report from the same PNG study examined whether a neonatal PCV7 dose might induce immune tolerance (59). In a comprehensive immuno-phenotypic analysis at 9 months of age, no differences in the quantity or quality of vaccine-specific T cell memory responses (including responses to CRM197, tetanus toxoid, and HBsAg) were found between the neonatal and infant vaccination groups. Hospitalization rates in the first month of life did not differ between children vaccinated with PCV at birth or not. Reviewing the data outlined in these two studies demonstrates that neonatal immunization with PCV7 is safe and not associated with immunologic tolerance $(56,58)$.

\section{CURRENT RESEARCH ON EARLY LIFE IMMUNIZATION}

\section{Enhancing Current Vaccines}

One approach to developing enhanced neonatal vaccines focuses on improving existing vaccines such as the live "selfadjuvanted" BCG vaccine. For example, the BCG-derivative VPM1002 expresses listeriolysin from Listeria monocytogenes designed to enhance MHC-I responses (60). In a phase II open label study comparison with conventional BCG-SSI in South African newborns $(n=48)$, VPM1002 demonstrated safety and immunogenicity with an increased proportion of CD8+ IL-17+ cells at 6 months post-vaccine. The authors speculate that although the significance of such cells is unknown, it is possible that they could contribute to more robust protection against TB and that larger studies are needed to assess this possibility.

\section{Development of Adjuvants for Early Life Immunization}

Another approach to enhancing vaccine responses in infants with "age-appropriate" immunity is the addition of adjuvantation systems to enhance vaccine immunogenicity and efficacy. PRR agonists such as mono-phosphoryl lipid A that activates TLR4, have been employed as vaccine adjuvants but the translational path for this approach must take into account that responses to PRR stimulation vary markedly with the age of a given individual (6). In developing adjuvant systems optimized to early life, there may be lessons to learn from live-attenuated vaccines currently in use. Examples from human in vitro studies using the BCG vaccine demonstrate this, including TLR2-mediated activation of neonatal NK cells to produce IFN $\gamma$; TLR9-mediated activation of pDCs for IFN $\gamma$; TLR2- and IFN-mediated activation of conventional DCs IL-12 p70 production and subsequent CD4+ T-cell Th1 polarization (61). Along these lines, TLR7/8 adjuvant-containing nanoparticles mimic effects of BCG on human neonatal monocyte-derived DCs in vitro and induce anti-mycobacterial T cells 
in humanized TLR8 mice in vivo (62). Addition of a TLR7/8 agonist adjuvant to PCV dramatically accelerated and enhanced responses to a birth dose, inducing pneumococcal-specific $\mathrm{Ab}$ titers far exceeding the CoP after a single dose (63). As with any vaccine development, safety will be front and center, and in this context, it is worth noting that the most commonly given neonatal vaccine, BCG, activates multiple PRRs including TLRs and is safely given to newborns across the globe. Although this does not necessarily imply that any TLR-stimulating approach would be safe, it does provide an important proof of concept that in certain settings and contexts, PRR activation including TLR-stimulation can be safe and effective approach to vaccine adjuvantation in human newborns.

\section{Mucosal Vaccine Development}

In designing new vaccines to be given in the neonatal period, consideration must be given to ideal characteristics (64). Mucosal vaccines, with their potential for needle-free delivery, are very attractive. A number of the pathogens causing severe disease in early infancy are mucosally transmitted [rotavirus, RSV, polio, non-typable Salmonella spp., enterotoxic Escherichia coli (ETEC)] and the positive experience with OPV provides proof of concept of mucosal vaccines in this age group. Mucosal vaccines could be administered by a number of routes (oral, nasal, conjunctival, rectal, and vaginal) with nasal and oral being the most practical and studied to date. Adjuvanted mucosally administered vaccines stimulate multiple types of immune responses, including secretory IgA Abs which prevent adhesion and invasion of pathogens, serum IgG neutralizing Abs, and a wide array of cell-mediated T cell responses (65). Of note, determining the correlate of immune protection for each vaccine is a challenge. The focus has historically been on neutralizing IgG antibodies, but increasingly, the role of antigen-specific IgA in serum and stool has been explored (66) with need for further standardization of these assays. A number of factors may influence the efficacy and immunogenicity of oral vaccines, particularly in early life, including the presence of pre-existing Abs, malnutrition, enteropathy, micronutrient deficiencies, and breast feeding, factors being studied in the context of OPV and rotavirus vaccines (67). Breast milk contains an array of protective molecules including specific Abs, oligosaccharides, glycoproteins, and receptor analogs, likely to prevent both pathogenic- and vaccine-strains of microorganisms binding to the intestinal wall. A study of Rotarix in infants comparing withholding or not withholding breastfeeding around the time of vaccine administration did not demonstrate an increase in anti-rotavirus IgA seroconversion (68). To the extent that these factors may limit responses to mucosal vaccines, neonates, who are unlikely to yet have malnutrition, enteropathy, and co-infections, may be more responsive to oral vaccines.

A number of oral vaccines with varying immunogenic and protective efficacies have been licensed including those directed against typhoid, rotavirus, polio, and cholera. Other targets include non-typable Salmonella, ETEC, Shigella, and adenovirus (64). Few to date have been studied in newborns, although diarrheal illness accounts for many of the deaths in infants $<6$ months of age, with rotavirus identified as a leading cause of dehydrating gastroenteritis, associated with $\sim 28 \%$ of diarrheal deaths (69). Two live-attenuated oral rotavirus vaccines, the pentavalent human-bovine rotavirus vaccine, RotaTeq, given as three oral doses a month apart; and monovalent human rotavirus vaccine Rotarix, given as two doses a month apart; were licensed in 2006. Protection against severe disease in high- and middle income countries is excellent for both (80-95\% efficacy). However, unfortunately, these rotavirus vaccines have been less efficacious in low-income regions where the need is far greater such as subSaharan Africa (46\% efficacy) and in Southern Asia (50\%) (70). Although indirect benefits of rotavirus vaccine, such as those realized via herd immunity and protection, have been described in high- and middle income countries, this has not been firmly established in low-income countries to date (71). Factors including breastmilk Abs, concurrent infections, or environmental enteropathy may interfere with the efficacy of the oral rotavirus vaccine (67). Administration of a birth dose could potentially ameliorate these concerns, as well as provide protection in the vulnerable gap when the most severe disease occurs, between birth and the protective response induced by a first dose given at 6 weeks of age (72). Furthermore, the risk of intussusception as a complication of rotavirus vaccine appears to follow an age-related pattern, supporting a neonatal schedule over an infant schedule for this vaccine (73). A phase IIa study of a monovalent human rotavirus vaccine $\mathrm{RV} 3-\mathrm{BB}$, including a neonatal dose, demonstrated a rotavirus IgA response rate of $11 \%$ with stool excretion of $13 \%$ after one dose (74). Overall immunogenicity following the non-neonatal schedule at 8,15 , and 24 weeks was $50 \%$ after two doses and $74 \%$ after 3 . A nested study within the trial examined the relationship between rotavirus-specific IgA in cord blood, colostrum and breast milk and infant serum IgA response and stool excretion and found no evidence of an association (75). Although these initial immunogenicity results are disappointing, with low uptake following the neonatal dose, neither RotaTeq nor Rotarix have been tested in the neonatal period such that further studies of rotavirus vaccines in the neonatal period are warranted.

A nasally delivered live-attenuated influenza vaccine (LAIV) has been effective in infants in protection from flu, but has not to date been tested in neonates. Infants between 6 and 12 months experienced relatively high rates of hospitalization in an RCT comparing inactivated vaccine and LAIV and consequently, LAIV has only been recommended in infants greater than 12 months of age (76). There is currently no available influenza vaccine for infants younger than 6 months, however, maternal immunization provides passive protection (77) and in a murine model, there was no evidence of maternal interference (78).

As a leading cause of morbidity and mortality in the neonatal and early infancy period, RSV is an ideal target for a mucosal vaccine (79). The RSV fusion (F) surface glycoprotein has been considered as one of the two major protective antigens for eliciting neutralizing Abs; a humanized monoclonal $\mathrm{Ab}$ specific to the $\mathrm{F}$ protein (Palivizumab), administered monthly to vulnerable infants during the RSV season, is efficacious in preventing severe disease, but not infection (80). Low levels of 
nasal RSV-specific IgA are a risk factor for RSV disease in adults (81), but as yet, although animal models have demonstrated neutralizing Abs in response to RSV vaccine candidates, specific IgA production has not been shown $(82,83)$. Animal models of live-attenuated vaccines provide grounds for optimism (84) and phase I studies of virus-vectored vaccines in adults have been promising (85), but virus-vectored RSV vaccines have not yet been trialed in infants. Development of vaccines based on the RSV pre-fusion protein, an antigenic target to which protective neutralizing Abs in human sera are directed (82), offers fresh avenues for RSV vaccine development with much to be learned regarding the potential of this antigen when administered in early life.

\section{Systems Biology}

Systems biology approaches have been applied to adults and older infants but thus far not to newborns (86). This deficiency is to soon change with the award of a NIH Human Immunology Project Consortium grant to employ systems vaccinology to study $\mathrm{HBV}$-induced molecular signatures in relation to $\mathrm{CoP}$-i.e., anti-HBsAg Ab responses. Led by the Expanded Program on Immunization Consortium, an affiliation of Boston Children's Hospital, Medical Research Council (UK)-Gambia, and the University of British Columbia, focused on application of systems biology to early life vaccinology, this study will leverage transcriptomics and proteomics to provide fresh insights into $\mathrm{HBV}$-induced protection in newborns. Among the novel systems biology approaches is "systems serology" that offers potential for much more precise understanding on the impact of vaccines at different ages measuring all aspects of the response, $\mathrm{Ab}$ avidity, titer, specific, and non-specific responses (87). This approach promises to provide deeper insight into the vaccine-induced humoral immune responses of distinct populations such as human newborns.

\section{In Vitro Modeling to Accelerate and De-Risk Early Life Vaccine Development}

Vaccine development is inherently costly, slow, and unfortunately beset by multiple failures. Current paradigms of vaccine development tend to presume that all populations will respond similarly to a given formulation and do not take species-specificity, genetic background, and age-specificity into account. Accordingly, many vaccine formulations fail or are less effective in vulnerable subpopulations such as the very young or elderly. In this context, human in vitro platforms that model age-specific vaccineinduced innate and adaptive immune responses as benchmarked to licensed vaccines offer the possibility of accelerating and derisking vaccine development $(9,88)$. Indeed age-specific human in vitro platforms such as newborn whole blood assays, dendritic cell arrays, and microphysiologic tissue constructs (89) have been successfully used to define novel biomarkers of vaccine adjuvanticity (90) and identify TLR7/8 agonists as adjuvants active in early life (63).

Overall these technical advances offer powerful new opportunities to inform, de-risk, and accelerate novel vaccine development for use in early life.

\section{INTEGRATION INTO CURRENT PROGRAM: CHALLENGES AND STRATEGIES}

Neonatal immunization carries tremendous potential but further expansion or enhancement of this approach will require both deeper mechanistic insight into how vaccines protect in early life as well as integration into the existing framework of public health, including maternal immunization programs. In response to the concerns of multiple professionals in the field of maternal immunization, the Brighton Collaboration was formed in 2000 , followed by the formation of the Global Alignment of Immunization safety Assessment in pregnancy (GAIA) to establish safety and efficacy standards in this area (91). A collection of case definitions and guidelines for data collection, analysis, and presentation of safety data in vaccine trials, relevant for neonates and infants, were published by global experts, largely in the context of maternal immunization, but these are also relevant and of value for monitoring safety of neonatal immunization studies (92). Whilst these definitions and guidelines can be amended for the use in neonatal immunization studies, the formation of an independent group is warranted to establish the framework for safe and efficacious neonatal immunization studies.

In 2010, to identify key topics and research gaps in the field and foster collaboration among investigators focusing on vaccinology and immune ontogeny, a workshop was organized by NIH (NIAID; Division of Allergy, Immunology, and Transplantation) and cosponsored by the Bill and Melinda Gates Foundation (93). Given recent technical and conceptual advances, and their potential to vastly transform the area of early life immunization, further workshops on optimizing early life immunization, including those individuals involved in regulation and safety assessments are warranted. The study populations for any ongoing vaccine trials should include not just healthy term infants, but a number of distinct populations, including preterm infants and those with immunodeficiencies, in particular HIV-exposed infected and uninfected [but not unaffected (94)], to ensure protection of all infants, especially the most vulnerable. Thoughtful design of studies, both of maternal and neonatal vaccines, will be essential to understand mechanisms underlying vaccinevaccine interactions, including interference. It is essential that whilst adopting the most advanced systems-based approaches, the data are standardized to allow comparison of sample sets from the same or different sites. Ongoing reassessment of infant immunization schedules will allow the development of more effective neonatal vaccine schedules.

\section{CONCLUSION}

Overall, neonatal immunization is a common practice across the globe, yet much can be done to optimize its beneficial impact. Taking advantage of pivotal opportunities to enhance this approach will require engagement with stakeholders, including government, funding agencies, and the general public, on: (a) the need for greater precision in our understanding 
of how current neonatal vaccines protect, the potential impact of the exact timing of administration in the neonatal period (i.e., first 28 days of life) and of vaccine-vaccine interactions, (b) assessing how maternal and neonatal immunization can be best integrated, and (c) leveraging modern tools including systems biology and human in vitro modeling to study the impact of immune ontogeny on vaccine responses thereby informing development of novel vaccines for use in early life against pathogens for which currently vaccines are inadequate (e.g., pertussis, TB, and influenza) or do not yet exist (e.g., RSV, HIV).

\section{AUTHOR CONTRIBUTIONS}

EW and OL outlined the initial manuscript drafts; EW, OL, PM, and DB all contributed to the final manuscript.

\section{REFERENCES}

1. Clemens J, Holmgren J, Kaufmann SHE, Mantovani A. Ten years of the global alliance for vaccines and immunization: challenges and progress. Nat Immunol (2010) 11:1069-72. doi:10.1038/ni1210-1069

2. Liu L, Oza S, Hogan D, Chu Y, Perin J, Zhu J, et al. Global, regional, and national causes of under-5 mortality in 2000-15: an updated systematic analysis with implications for the sustainable development goals. Lancet (2016) 388:3027-35. doi:10.1016/S0140-6736(16)31593-8

3. Marchant A, Sadarangani M, Garand M, Dauby N, Verhasselt V, Pereira L, et al. Maternal immunisation: collaborating with mother nature. Lancet Infect Dis (2017) 17:e197-208. doi:10.1016/S1473-3099(17)30229-3

4. Kollmann TR, Crabtree J, Rein-Weston A, Blimkie D, Thommai F, Wang XY, et al. Neonatal innate TLR-mediated responses are distinct from those of adults. J Immunol (2009) 183:7150-60. doi:10.4049/jimmunol.0901481

5. Levy O, Goriely S, Kollmann TR. Immune response to vaccine adjuvants during the first year of life. Vaccine (2013) 31:2500-5. doi:10.1016/j.vaccine. 2012.10.016

6. Kollmann TR, Levy O, Montgomery RR, Goriely S. Innate immune function by toll-like receptors: distinct responses in newborns and the elderly. Immunity (2012) 37:771-83. doi:10.1016/j.immuni.2012.10.014

7. Miller JE, Hammond GC, Strunk T, Moore HC, Leonard H, Carter KW, et al. Association of gestational age and growth measures at birth with infection-related admissions to hospital throughout childhood: a population-based, data-linkage study from Western Australia. Lancet Infect Dis (2016) 16:952-61. doi:10.1016/S1473-3099(16)00150-X

8. Aaby P, Kollmann TR, Benn CS. Nonspecific effects of neonatal and infant vaccination: public-health, immunological and conceptual challenges. Nat Immunol (2014) 15:895-9. doi:10.1038/ni.2961

9. Dowling DJ, Levy O. Ontogeny of early life immunity. Trends Immunol (2014) 35:299-310. doi:10.1016/j.it.2014.04.007

10. Kollmann TR, Kampmann B, Mazmanian SK, Marchant A, Levy O. Protecting the newborn and young infant from infectious diseases: lessons from immune ontogeny. Immunity (2017) 46:350-63. doi:10.1016/j.immuni.2017. 03.009

11. Pettengill MA, van Haren SD, Levy O. Soluble mediators regulating immunity in early life. Front Immunol (2014) 5:457. doi:10.3389/fimmu.2014.00457

12. Namdar A, Koleva P, Shahbaz S, Strom S, Gerdts V, Elahi S. CD71(+) erythroid suppressor cells impair adaptive immunity against Bordetella pertussis. Sci Rep (2017) 7:7728. doi:10.1038/s41598-017-07938-7

13. Mohr E, Siegrist C-A. Vaccination in early life: standing up to the challenges. Curr Opin Immunol (2016) 41:1-8. doi:10.1016/j.coi.2016.04.004

14. Vekemans J, Amedei A, Ota MO, D’Elios MM, Goetghebuer T, Ismaili J, et al. Neonatal Bacillus Calmette-Guérin vaccination induces adult-like IFNgamma production by CD4+ Tlymphocytes. Eur J Immunol (2001) 31:1531-5. doi:10.1002/1521-4141(200105)31:5<1531:AID-IMMU1531>3.0.CO;2-1

\section{FUNDING}

OL's laboratory has been supported by Grand Challenges Explorations (OPP1035192) and Global Health (OPPGH5284) Awards from the Bill \& Melinda Gates Foundation as well as US National Institutes of Health National Institutes of Allergy and Infectious Diseases Infant Immunity program grant 1R01AI100135-01, Molecular Mechanisms of Combination Adjuvants U01AI124284-01, and Adjuvant Discovery Program contract HHSN272201400052C, Human Immunology Project Consortium Award U19AI118608, an internal Boston Children's Hospital award to the Precision Vaccines Program, as well as sponsored support from 3M Drug Delivery Systems, MedImmune and VentiRx Pharmaceuticals DG's lab receives research support from vaccine manufacturers including GSK. He is an occasional member of vaccine advisory boards for GSK, Merck, and Sanofi Pasteur. He is an NIHR Senior Investigator.

15. Shann F. Substantial benefits from finding the most effective BCG strain. Lancet Respir Med (2016) 4:e35. doi:10.1016/S2213-2600(16)30108-4

16. Saadatian-Elahi M, Aaby P, Shann F, Netea MG, Levy O, Louis J, et al. Heterologous vaccine effects. Vaccine (2016) 34:3923-30. doi:10.1016/j. vaccine.2016.06.020

17. Morra ME, Kien ND, Elmaraezy A, Abdelaziz OAM, Elsayed AL, Halhouli O, et al. Early vaccination protects against childhood leukemia: a systematic review and meta-analysis. Sci Rep (2017) 7:15986. doi:10.1038/s41598-017-16067-0

18. Rousseau MC, Zein EM, Conus F, Legault L, Parent ME. Bacillus Calmette-Guérin (BCG) vaccination in infancy and risk of childhood diabetes. Paediatr Perinat Epidemiol (2016) 30:141-8. doi:10.1111/ ppe. 12263

19. Thøstesen LM, Kjaergaard J, Pihl GT, Birk NM, Nissen TN, Aaby P, et al. Neonatal BCG vaccination and atopic dermatitis before 13 months of age: a randomized clinical trial. Allergy (2018) 73:498-504. doi:10.1111/all.13314

20. Plotkin SA, Gilbert PB. Nomenclature for immune correlates of protection after vaccination. Clin Infect Dis (2012) 54:1615-7. doi:10.1093/cid/cis238

21. Hatherill M, Tait D, Mcshane $\mathrm{H}$. Clinical testing of tuberculosis vaccine candidates. Microbiol Spectr (2016) 4:1-18. doi:10.1128/microbiolspec. TBTB2-0015-2016

22. Favorov M, Ali M, Tursunbayeva A, Aitmagambetova I, Kilgore P, Ismailov S, et al. Comparative tuberculosis (TB) prevention effectiveness in children of Bacillus Calmette-Guérin (BCG) vaccines from different sources, Kazakhstan. PLoS One (2012) 7:e32567. doi:10.1371/journal.pone.0032567

23. Kleinnijenhuis J, Oosting M, Joosten LAB, Netea MG, van Crevel R. Innate immune recognition of Mycobacterium tuberculosis. Clin Dev Immunol (2011) 2011:405310. doi:10.1155/2011/405310

24. Wynn JL, Scumpia PO, Winfield RD, Delano MJ, Kelly-Scumpia K, Barker $\mathrm{T}$, et al. Defective innate immunity predisposes murine neonates to poor sepsis outcome but is reversed by TLR agonists. Blood (2008) 112:1750-8. doi:10.1182/blood-2008-01-130500

25. Netea MG, Joosten LAB, Latz E, Mills KHG, Natoli G, Stunnenberg HG, et al. Trained immunity: a program of innate immune memory in health and disease. Science (2016) 352:aaf1098. doi:10.1126/science.aaf1098

26. Strategic Advisory Group of Experts (SAGE). WHO Weekly Epidemiological Record. (Vol. 21). (2014). p. 221-36. Available from: http://www.who.int/ wer/2014/wer8921.pdf?ua=1, http://www.who.int/immunization/sage/ meetings/2014/april/en/

27. Levy O, Netea MG. Innate immune memory: implications for development of pediatric immunomodulatory agents and adjuvanted vaccines. Pediatr Res (2014) 75:184-8. doi:10.1038/pr.2013.214

28. Committee on Infectious Diseases, Committee on Fetus and Newborn. Elimination of perinatal hepatitis B: providing the first vaccine dose within 24 hours of birth. Pediatrics (2017) 140:e20171870. doi:10.1542/peds.2017-1870

29. van Montfoort N, van der Aa E, van den Bosch A, Brouwers H, Vanwolleghem T, Janssen HLA, et al. Hepatitis B virus surface antigen activates myeloid 
dendritic cells via a soluble CD14-dependent mechanism. J Virol (2016) 90:6187-99. doi:10.1128/JVI.02903-15

30. Gelinas L, Abu-Raya B, Ruck C, Cai B, Kollmann TR. Hepatitis B virus vaccine-induced cell-mediated immunity correlates with humoral immune response following primary vaccination during infancy. Immunohorizons (2017) 1:42-52. doi:10.4049/immunohorizons.1700015

31. Yan K, Cai W, Cao F, Sun H, Chen S, Xu R, et al. Genetic effects have a dominant role on poor responses to infant vaccination to hepatitis B virus. J Hum Genet (2013) 58:293-7. doi:10.1038/jhg.2013.18

32. Ryckman KK, Morken N-H, White MJ, Velez DR, Menon R, Fortunato SJ, et al. Maternal and fetal genetic associations of PTGER3 and PON1 with preterm birth. PLoS One (2010) 5:e9040. doi:10.1371/journal.pone.0009040

33. World Health Organization. Polio vaccines: WHO position paper, March 2016-recommendations. Vaccine (2017) 35:1197-9. doi:10.1016/j. vaccine.2016.11.017

34. Mateen FJ, Shinohara RT, Sutter RW. Oral and inactivated poliovirus vaccines in the newborn: a review. Vaccine (2013) 31:2517-24. doi:10.1016/j. vaccine.2012.06.020

35. Lund N, Andersen A, Hansen ASK, Jepsen FS, Barbosa A, Biering-Sørensen S, et al. The effect of oral polio vaccine at birth on infant mortality: a randomized trial. Clin Infect Dis (2015) 61:1504-11. doi:10.1093/cid/civ617

36. Fish EN, Flanagan KL, Furman D, Klein SL, Kollmann TR, Jeppesen DL, et al. Changing oral vaccine to inactivated polio vaccine might increase mortality. Lancet (2016) 387:1054-5. doi:10.1016/S0140-6736(16)00661-9

37. Halsey N, Galazka A. The efficacy of DPT and oral poliomyelitis immunization schedules initiated from birth to 12 weeks of age. Bull World Health Organ (1985) 63:1151-69.

38. Provenzano RW, Wetterlow LH, Sullivan CL. Immunization and antibody response in the newborn infant. I. Pertussis inoculation within twenty-four hours of birth. N Engl J Med (1965) 273:959-65. doi:10.1056/ NEJM196510282731804

39. Baraff LJ, Leake RD, Burstyn DG, Payne T, Cody CL, Manclark CR, et al. Immunologic response to early and routine DTP immunization in infants. Pediatrics (1984) 73:37-42.

40. Belloni C, Silvestri AD, Tinelli C, Avanzini MA, Marconi M, Strano F, et al. Immunogenicity of a three-component acellular pertussis vaccine administered at birth. Pediatrics (2003) 111:1042-5. doi:10.1542/peds.111.5.1042

41. Knuf M, Schmitt H-J, Wolter J, Schuerman L, Jacquet J-M, Kieninger D, et al. Neonatal vaccination with an acellular pertussis vaccine accelerates the acquisition of pertussis antibodies in infants. J Pediatr (2008) 152:655-60,660. e1. doi:10.1016/j.jpeds.2007.09.034

42. Halasa NB, O'Shea A, Shi JR, LaFleur BJ, Edwards KM. Poor immune responses to a birth dose of diphtheria, tetanus, and acellular pertussis vaccine. J Pediatr (2008) 153:327-32. doi:10.1016/j.jpeds.2008.03.011

43. Wood N, McIntyre P, Marshall H, Roberton D. Acellular pertussis vaccine at birth and one month induces antibody responses by two months of age. Pediatr Infect Dis J (2010) 29:209-15. doi:10.1097/INF.0b013e3181bc98d5

44. Wood N, Marshall H, White OJ, Holt PG, McIntyre P. Antibody and cell-mediated immunity to pertussis 4 years after monovalent acellular pertussis vaccine at birth. Pediatr Infect Dis J (2014) 33:511-7. doi:10.1097/ INF.0000000000000246

45. Knuf M, Schmitt H-J, Jacquet J-M, Collard A, Kieninger D, Meyer CU, et al. Booster vaccination after neonatal priming with acellular pertussis vaccine. J Pediatr (2010) 156:675-8. doi:10.1016/j.jpeds.2009.12.019

46. Maertens K, Tran TMP, Hens N, Van Damme P, Leuridan E. Effect of prepregnancy pertussis vaccination in young infants. J Infect Dis (2017) 215:1855-61. doi:10.1093/infdis/jix176

47. Englund JA, Anderson EL, Reed GF, Decker MD, Edwards KM, Pichichero ME, et al. The effect of maternal antibody on the serologic response and the incidence of adverse reactions after primary immunization with acellular and whole-cell pertussis vaccines combined with diphtheria and tetanus toxoids. Pediatrics (1995) 96:580-4.

48. Gu X-X, Plotkin SA, Edwards KM, Sette A, Mills KHG, Levy O, et al. Waning immunity and microbial vaccines-workshop of the national institute of allergy and infectious diseases. Clin Vaccine Immunol (2017) 24:1-15. doi:10.1128/ CVI.00034-17

49. Latz E, Franko J, Golenbock DT, Schreiber JR. Haemophilus influenzae type b-outer membrane protein complex glycoconjugate vaccine induces cytokine production by engaging human toll-like receptor 2 (TLR2) and requires the presence of TLR2 for optimal immunogenicity. J Immunol (2004) 172:2431-8. doi:10.4049/jimmunol.172.4.2431

50. Kurikka S, Käyhty H, Peltola H, Saarinen L, Eskola J, Mäkelä PH. Neonatal immunization: response to Haemophilus influenzae type b-tetanus toxoid conjugate vaccine. Pediatrics (1995) 95:815-22.

51. Lieberman JM, Greenberg DP, Wong VK, Partridge S, Chang SJ, Chiu CY, et al. Effect of neonatal immunization with diphtheria and tetanus toxoids on antibody responses to Haemophilus influenzae type b conjugate vaccines. J Pediatr (1995) 126:198-205. doi:10.1016/S0022-3476(95)70545-7

52. McVERNON J, HOWARD AJ, SLACK MPE, Ramsay ME. Long-term impact of vaccination on Haemophilus influenzae type b (Hib) carriage in the United Kingdom. Epidemiol Infect (2004) 132:765-7. doi:10.1017/s0950268804002122

53. Adegbola RA, Mulholland EK, Secka O, Jaffar S, Greenwood BM. Vaccination with a Haemophilus influenzae type b conjugate vaccine reduces oropharyngeal carriage of $H$. influenzae type b among Gambian children. J Infect Dis (1998) 177:1758-61. doi:10.1086/517440

54. Gkentzi D, Slack MPE, Ladhani SN. The burden of nonencapsulated Haemophilus influenzae in children and potential for prevention. Curr Opin Infect Dis (2012) 25:266-72. doi:10.1097/QCO.0b013e32835310a4

55. MacNeil JR, Cohn AC, Farley M, Mair R, Baumbach J, Bennett N, et al. Current epidemiology and trends in invasive Haemophilus influenzae disease-United States, 1989-2008. Clin Infect Dis (2011) 53:1230-6. doi:10.1093/cid/cir735

56. Pomat WS, van den Biggelaar AHJ, Phuanukoonnon S, Francis J, Jacoby P, Siba PM, et al. Safety and immunogenicity of neonatal pneumococcal conjugate vaccination in Papua New Guinean children: a randomised controlled trial. PLoS One (2013) 8:e56698. doi:10.1371/journal. pone. 0056698

57. Scott JAG, Ojal J, Ashton L, Muhoro A, Burbidge P, Goldblatt D. Pneumococcal conjugate vaccine given shortly after birth stimulates effective antibody concentrations and primes immunological memory for sustained infant protection. Clin Infect Dis (2011) 53:663-70. doi:10.1093/cid/cir444

58. Aho C, Michael A, Yoannes M, Greenhill A, Jacoby P, Reeder J, et al. Limited impact of neonatal or early infant schedules of 7-valent pneumococcal conjugate vaccination on nasopharyngeal carriage of Streptococcus pneumoniae in Papua New Guinean children: a randomized controlled trial. Vaccine Rep (2016) 6:36-43. doi:10.1016/j.vacrep.2016.08.002

59. van den Biggelaar AHJ, Pomat W, Bosco A, Phuanukoonnon S, Devitt CJ, Nadal-Sims MA, et al. Pneumococcal conjugate vaccination at birth in a high-risk setting: no evidence for neonatal T-cell tolerance. Vaccine (2011) 29:5414-20. doi:10.1016/j.vaccine.2011.05.065

60. Loxton AG, Knaul JK, Grode L, Gutschmidt A, Meller C, Eisele B, et al. Safety and immunogenicity of the recombinant Mycobacterium bovis BCG vaccine VPM1002 in HIV-unexposed newborn infants in South Africa. Clin Vaccine Immunol (2017) 24:e439-416. doi:10.1128/CVI.00439-16

61. Kativhu CL, Libraty DHA. Model to explain how the Bacille Calmette Guérin (BCG) vaccine drives interleukin-12 production in neonates. PLoS One (2016) 11:e0162148. doi:10.1371/journal.pone.0162148

62. Dowling DJ, Scott EA, Scheid A, Bergelson I, Joshi S, Pietrasanta C, et al. Toll-like receptor 8 agonist nanoparticles mimic immunomodulating effects of the live BCG vaccine and enhance neonatal innate and adaptive immune responses. J Allergy Clin Immunol (2017) 140:1339-50. doi:10.1016/j. jaci.2016.12.985

63. Dowling DJ, Van Haren SD, Scheid A, Bergelson I, Kim D, Mancuso CJ, et al. TLR7/8 adjuvant overcomes newborn hyporesponsiveness to pneumococcal conjugate vaccine at birth. JCI Insight (2017) 2:e91020. doi:10.1172/jci. insight. 91020

64. Levine MM. "IDEAL" vaccines for resource poor settings. Vaccine (2011) 29(Suppl 4):D116-25. doi:10.1016/j.vaccine.2011.11.090

65. Neutra MR, Kozlowski PA. Mucosal vaccines: the promise and the challenge. Nat Rev Immunol (2006) 6:148-58. doi:10.1038/nri1777

66. Wright PF, Connor RI, Wieland-Alter WF, Hoen AG, Boesch AW, Ackerman $\mathrm{ME}$, et al. Vaccine-induced mucosal immunity to poliovirus: analysis of cohorts from an open-label, randomised controlled trial in Latin American infants. Lancet Infect Dis (2016) 16:1377-84. doi:10.1016/S1473-3099(16) 30169-4

67. Sindhu KNC, Cunliffe N, Peak M, Turner M, Darby A, Grassly N, et al. Impact of maternal antibodies and infant gut microbiota on the immunogenicity of rotavirus vaccines in African, Indian and European infants: protocol for 
a prospective cohort study. BMJ Open (2017) 7:e016577. doi:10.1136/ bmjopen-2017-016577

68. Ali A, Kazi AM, Cortese MM, Fleming JA, Moon S, Parashar UD, et al. Impact of withholding breastfeeding at the time of vaccination on the immunogenicity of oral rotavirus vaccine - a randomized trial. PLoS One (2015) 10:e0127622. doi:10.1371/journal.pone.0127622

69. Lanata CF, Fischer-Walker CL, Olascoaga AC, Torres CX, Aryee MJ, Black RE, et al. Global causes of diarrheal disease mortality in children $<5$ years of age: a systematic review. PLoS One (2013) 8:e72788. doi:10.1371/journal. pone. 0072788

70. Lamberti LM, Ashraf S, Walker CLF, Black RE. A systematic review of the effect of rotavirus vaccination on diarrhea outcomes among children younger than 5 years. Pediatr Infect Dis J (2016) 35:992-8. doi:10.1097/ INF.0000000000001232

71. Bennett A, Bar-Zeev N, Cunliffe NA. Measuring indirect effects of rotavirus vaccine in low income countries. Vaccine (2016) 34:4351-3. doi:10.1016/j. vaccine.2016.07.001

72. Mathew A, Rao PSS, Sowmyanarayanan TV, Kang G. Severity of rotavirus gastroenteritis in an Indian population: report from a 3 year surveillance study. Vaccine (2014) 32(Suppl 1):A45-8. doi:10.1016/j.vaccine.2014.03.038

73. Simonsen L, Viboud C, Elixhauser A, Taylor RJ, Kapikian AZ. More on RotaShield and intussusception: the role of age at the time of vaccination. J Infect Dis (2005) 192(Suppl 1):S36-43. doi:10.1086/431512

74. Bines JE, Danchin M, Jackson P, Handley A, Watts E, Lee KJ, et al. Safety and immunogenicity of RV3-BB human neonatal rotavirus vaccine administered at birth or in infancy: a randomised, double-blind, placebo-controlled trial. Lancet Infect Dis (2015) 15:1389-97. doi:10.1016/s1473-3099(15)00227-3

75. Chen M-Y, Kirkwood CD, Bines J, Cowley D, Pavlic D, Lee KJ, et al. Rotavirus specific maternal antibodies and immune response to RV3-BB neonatal rotavirus vaccine in New Zealand. Hum Vaccin Immunother (2017) 13:1126-35. d oi:10.1080/21645515.2016.1274474

76. Belshe RB, Edwards KM, Vesikari T, Black SV, Walker RE, Hultquist M, et al. Live attenuated versus inactivated influenza vaccine in infants and young children. N Engl J Med (2007) 356:685-96. doi:10.1056/NEJMoa065368

77. Chu HY, Englund JA. Maternal immunization. Clin Infect Dis (2014) 59:560-8. doi:10.1093/cid/ciu327

78. van der Lubbe JEM, Vreugdenhil J, Damman S, Vaneman J, Klap J, Goudsmit $\mathrm{J}$, et al. Maternal antibodies protect offspring from severe influenza infection and do not lead to detectable interference with subsequent offspring immunization. Virol J (2017) 14:123. doi:10.1186/s12985-017-0787-4

79. Shi T, McAllister DA, O’Brien KL, Simões EAF, Madhi SA, Gessner BD, et al. Global, regional, and national disease burden estimates of acute lower respiratory infections due to respiratory syncytial virus in young children in 2015: a systematic review and modelling study. Lancet (2017) 390:946-58. doi:10.1016/S0140-6736(17)30938-8

80. Ruckwardt TJ, Morabito KM, Graham BS. Determinants of early life immune responses to RSV infection. Curr Opin Virol (2016) 16:151-7. doi:10.1016/j. coviro.2016.01.003

81. Walsh EE, Falsey AR. Humoral and mucosal immunity in protection from natural respiratory syncytial virus infection in adults. J Infect Dis (2004) 190:373-8. doi:10.1086/421524

82. Graham BS. Vaccine development for respiratory syncytial virus. Curr Opin Virol (2017) 23:107-12. doi:10.1016/j.coviro.2017.03.012
83. Yang K, Varga SM. Mucosal vaccines against respiratory syncytial virus. Curr Opin Virol (2014) 6:78-84. doi:10.1016/j.coviro.2014.03.009

84. Gerretsen HE, Sande CJ. Development of respiratory syncytial virus (RSV) vaccines for infants. JInfect (2017) 74(Suppl 1):S143-6. doi:10.1016/ S0163-4453(17)30205-0

85. Green CA, Scarselli E, Sande CJ, Thompson AJ, de Lara CM, Taylor KS, et al. Chimpanzee adenovirus- and MVA-vectored respiratory syncytial virus vaccine is safe and immunogenic in adults. Sci Transl Med (2015) 7:ra126-300. doi:10.1126/scitranslmed.aac5745

86. Amenyogbe N, Levy O, Kollmann TR. Systems vaccinology: a promise for the young and the poor. Philos Trans R Soc Lond B Biol Sci (2015) 370:2014034020140340. doi:10.1098/rstb.2014.0340

87. Chung AW, Kumar MP, Arnold KB, Yu WH, Schoen MK, Dunphy LJ, et al. Dissecting polyclonal vaccine-induced humoral immunity against HIV using systems serology. Cell (2015) 163:988-98. doi:10.1016/j. cell.2015.10.027

88. Sanchez-Schmitz G, Levy O. Development of newborn and infant vaccines. Sci Transl Med (2011) 3:s27-90. doi:10.1126/scitranslmed.3001880

89. Sanchez-Schmitz G, Stevens C, Baecher-Allan C, Levy O. A novel human neonatal tissue construct (NTC) models age-specific immune responses to bacille Calmette-Guérin (BCG) vaccine. Abstracts from the 99th Annual Meeting of the American Society of Immunologists (Boston) Journal of Immunology. Vol. 188. Boston (2012); p. 166.27.

90. Oh D-Y, Dowling DJ, Ahmed S, Choi H, Brightman S, Bergelson I, et al Adjuvant-induced human monocyte secretome profiles reveal adjuvant- and age-specific protein signatures. Mol Cell Proteomics (2016) 15:1877-94. doi:10.1074/mcp.M115.055541

91. Bonhoeffer J, Kohl K, Chen R, Duclos P, Heijbel H, Heininger U, et al. The brighton collaboration: addressing the need for standardized case definitions of adverse events following immunization (AEFI). Vaccine (2002) 21:298-302. doi:10.1016/S0264-410X(02)00449-8

92. Vergnano S, Buttery J, Cailes B, Chandrasekaran R, Chiappini E, Clark E, et al. Neonatal infections: case definition and guidelines for data collection, analysis, and presentation of immunisation safety data. Vaccine (2016) 34:6038-46. doi:10.1016/j.vaccine.2016.03.046

93. PrabhuDas M, Adkins B, Gans H, King C, Levy O, Ramilo O, et al. Challenges in infant immunity: implications for responses to infection and vaccines. Nat Immunol (2011) 12:189-94. doi:10.1038/ni0311-189

94. Dauby N, Goetghebuer T, Kollmann TR, Levy J, Marchant A. Uninfected but not unaffected: chronic maternal infections during pregnancy, fetal immunity, and susceptibility to postnatal infections. Lancet Infect Dis (2012) 12:330-40. doi:10.1016/S1473-3099(11)70341-3

Conflict of Interest Statement: The authors declare that the research was conducted in the absence of any commercial or financial relationships that could be construed as a potential conflict of interest.

Copyright $\odot 2018$ Whittaker, Goldblatt, McIntyre and Levy. This is an open-access article distributed under the terms of the Creative Commons Attribution License (CC $B Y)$. The use, distribution or reproduction in other forums is permitted, provided the original author(s) and the copyright owner are credited and that the original publication in this journal is cited, in accordance with accepted academic practice. No use, distribution or reproduction is permitted which does not comply with these terms. 